Received 00th January 20xx, Accepted 00th January 20xx DOI: $10.1039 / x 0 x \times 00000 x$

\title{
Encapsulation of lipophilic fragrance by Polymerization of the intermediate aqueous phase of an oil-in-water-in-oil (0/W/O) double emulsion
}

\author{
Margot Stasse, ${ }^{a-b-c}$ Tiphaine Ribaut $^{c}$, Véronique Schmitt ${ }^{* b}$ and Valérie Héroguez ${ }^{* a}$
}

\begin{abstract}
This paper deals with the encapsulation of lipophilic compounds in a double oil-in-water-in-oil $(\mathrm{O} / \mathrm{W} / \mathrm{O})$ emulsion. Such encapsulation is of special interest in cosmetics where fragrance is generally a mixture of various lipophilic reactive species. One of the most important challenges in encapsulation is to prevent the immediate leakage of the encapsulated species. In this context, our strategy was to develop capsules by separating the compartments containing the compounds to encapsulate and the locus of polymerization. This is achieved by polymerizing the water phase of an $\mathrm{O} / \mathrm{W} / \mathrm{O}$ double emulsion. In a first part, the formulation and the free radical polymerization of reverse (W/O) simple emulsions with various hydrophilic monomers were studied. Two monomers have been selected, Methacrylamide (MAM) and Oligoethyleneglycolmethacrylate (OEGMA). In a second part, double O/W/O emulsions were formulated and several compositions for which only multiple droplets have been observed were identified. Then the aqueous phase was polymerized. Conversion of monomers was not complete, although the polymerizations occurred without coagulum.
\end{abstract}

\section{Introduction}

Encapsulation of lipophilic components exhibits several advantages. First of all, it allows the protection of the encapsulated species from the oxidation or deterioration, it also permits to spatially and kinetically control the release of the encapsulated compounds i.e. over a given time and/or on a specific area ${ }^{1,2}$. Encapsulation appeared in 1957 for carbonless paper $^{3}$. Nowadays, encapsulation is present everywhere as in pharmaceutical $^{4}$, agro-food ${ }^{5}$, phyto-sanitary ${ }^{6}$ industries or cosmetics and fragrance applications ${ }^{7,8}$. Two main categories of capsules are described in literature ${ }^{2}$ : the core-shell and the matrix capsules. In the latter, the active ingredients are integrated within the matrix capsules. Various synthesizing pathways are described to elaborate all kinds of capsules: Layerby-Layer ${ }^{9,10}$, solvent evaporation ${ }^{11}$, interfacial polycondensation ${ }^{12,13}$ or suspension polymerization ${ }^{14,15}$. However, for most of these techniques, monomer is located in the same phase as the encapsulated species. In the specific case of fragrance, the encapsulated species is a complex mixture of different molecules such as ketones, alcohols, aldehydes, amines, nitriles, aromatics, alkenes or conjugated alkenes. These compounds possess reactive functions enable to react

\footnotetext{
a. Laboratoire de Chimie des Polymères Organiques, CNRS, UMR 5629, Bordeaux, 16 Avenue Pey-Berland, F-33607 Pessac, France.

b. Centre de Recherche Paul Pascal, UMR 5031 University of Bordeaux CNRS 115 avenue du Dr Albert Schweitzer 33600 Pessac, France.

. Takasago Europe Perfumery Laboratory 12 rue Torricelli 75017 PARIS, France.

+ Footnotes relating to the title and/or authors should appear here.

Electronic Supplementary Information (ESI) available: [details of any supplementary information available should be included here]. See DOI: 10.1039/x0xx00000x
}

during the polymerization step necessary to elaborate the capsules. Due to their reactive groups, the fragrance molecules can be altered, therefore modifying be the olfactory notes.

To avoid these issues, we propose a strategy, which allows the compartmentalization of the polymerization and the fragrance storage. First, a double emulsion of fragrance-in-water-in-oil is formulated and then the intermediate aqueous phase is polymerized to create a polymeric barrier between the fragrance and the external oil phase. Likewise, we aim at obtaining capsules able to release the fragrance by diffusion through the polymeric barrier with a tunable kinetics of release that can be controlled by changing the monomers.

In a previous study ${ }^{16}$, we determined the best conditions to obtain a double emulsion of fragrance-in-water-in cosmetic oil without monomer in the aqueous phase. Several compositions, leading to stable enough double emulsions, have been identified. Herein, we focus on the formulation and polymerization of these double emulsions containing monomer inside the water phase. Double emulsions are complex systems containing two antagonistic stabilizers (a more hydrophilic surfactant (HS) to stabilize the oil-in-water emulsion and a more lipophilic one (LS) to stabilize the reverse water-in-oil emulsion). Consequently, each additional compound may interact with the established formula. Therefore, in the present paper we chose a progressive approach. In a first step, the reverse emulsion containing monomers inside the water phase will be studied in terms of feasibility and stability. This will allow selecting the most interesting monomers for the study in terms of stability and emulsions' size. In a second step, the obtained reverse emulsion will be polymerized. In the third step, double emulsions will be formulated with the previously selected monomers. In the last step, the double emulsion will be 
polymerized. The results will allow determining whether the fragrance present as droplets inside the water phase has an effect on the polymerization.

\section{Experimental section}

\section{1-Chemicals}

All the reagents were purchased and used without further purification. For polymerization, monomers and water were degassed by nitrogen bubbling during 1 hour. Polyglycerol polyricinoleate 4150 (PGPR) was graciously provided by Paalsgard (molar mass of 3000 g.mol ${ }^{-1}$ and $\mathrm{HLB}=1.5$ ) and Tergitol 15-S-12 was purchased from Sigma-Aldrich (Polyglycol ether, CAS number 68131-40-8, molar mass $=760 \mathrm{~g} \cdot \mathrm{mol}^{-1}$ and HLB=14.5). The external isopropyl myristaste oil (IPM, density equal to $0.850 \mathrm{~g} \cdot \mathrm{mL}^{-1}$ ) is widely used in perfumery. It was provided by Takasago European Perfumery Laboratory (T.E.P.L). Two different fragrances hereafter named $A$ and $B$ composed of 10 and 13 molecules for fragrance $A$ and $B$ respectively have been specially formulated by T.E.P.L.. Their complete compositions are given in Supplementary Information SI1. The fragrance $A$ is composed with molecules which do not possess alkene functions. The fragrance $B$, in contrast, is largely composed of molecules with alkene functions.

Their physical properties are summarized in Table 1. All other reactants were purchased from Sigma Aldrich: Hydroxyethylmethacrylate (98\%)(HEMA), Hydroxy Oligoethyleneglycolmethacrylate (OEGMA) with $\mathrm{M}_{\mathrm{n}}=360 \mathrm{~g} \cdot \mathrm{mol}-1$ (contains 500-800 ppm of monomethyl ether hydroquinone), Methacrylamide (MAM) (98\%), $\mathrm{NaCl}$ (99\%) and Potassium persulfate (KPS) (99\%). Milli-Q water was used in all emulsion preparations.

The relative solubility of the fragrance in water and in octanol, LogP, is defined as:

$$
\log \mathrm{P}=\log \left(\frac{\mathrm{C}_{\text {octanol }}}{\mathrm{C}_{\text {water }}}\right)
$$

where $P$ is the partition coefficient between water and octanol, $\mathrm{C}_{\text {octanol }}$ and $\mathrm{C}_{\text {water }}$ are the concentration of the considered species in octanol and water respectively. A lipophilic species, more soluble in octanol (resp. hydrophilic species, more soluble in water) than in water (resp. than in octanol) leads to a high and positive (resp low and negative) value of LogP. If LogP is equal to zero, then the molecule exhibits the same affinity for both solvents. In the present case, as the fragrance is a mixture of molecules, the molar weighted average value of LogP is calculated $^{17}$. For both fragrances, the values are positive, meaning a higher solubility in octanol than in water. Moreover, the LogP values of the two fragrances are very close to each other.

Table 1: Physical properties of each fragrance

\begin{tabular}{|l|c|c|c|c|}
\hline Properties & LogP & $\begin{array}{c}\text { Refractive } \\
\text { Index }\end{array}$ & Density & $\begin{array}{c}\sigma \\
(\mathrm{mN} / \mathrm{m})\end{array}$ \\
\hline Fragrance A & 3.38 & 1.454 & 0.869 & 21.8 \\
\hline Fragrance B & 3.52 & 1.475 & 0.887 & 34.6 \\
\hline
\end{tabular}

$\sigma=$ Interfacial tension against $\mathrm{H}_{2} \mathrm{O}$

\section{2-Emulsion size characterization}

The emulsions drop size distributions were measured using static light scattering by means of a Malvern Mastersizer MS2000 granulometer. For simple emulsions, the collected scattered intensity as a function of the angle was transformed into the size distribution using the Mie theory. The emulsions were diluted prior to measurements to avoid multiple scattering effects. We checked by optical microscopy that dilution required for static light scattering did not alter the droplet size. The refractive indices were measured using an Abbé refractometer, they were equal to 1.333 for water or brine (no significant difference has been detected for $0.1 \mathrm{M} \mathrm{NaCl}$ solution compared to water), 1.434 for IPM and to 1.454 and 1.475 for fragrances $A$ and $B$ respectively.

For the multiple emulsions, since oily globules contained water droplets, they were not optically homogeneous anymore. Therefore, Mie theory could not be used anymore. We chose the simplified Fraunhofer theory where drops are approximated by opaque discs and the principles of geometrical optics apply ${ }^{18}$.

For all types of emulsions, the granulometer gave the volume average diameter $d$ and the polydispersity index $P$, defined by equation (2):

$$
\mathrm{d}=\frac{\sum_{i} N_{i} D_{i}^{4}}{\sum_{i} N_{i} D_{i}^{3}} \quad \mathrm{P}=\frac{1}{D_{m}} \frac{\sum_{i} N_{i} D_{i}^{3}\left|D_{m}-D_{i}\right|}{\sum_{i} N_{i} D_{i}^{3}}
$$

Where $D_{i}, N_{i}$ and $D_{m}$ are the diameter of the drops, the number of the drops and the median diameter respectively.

\section{3-Preparation of the reverse emulsion}

In the following, all the inverted emulsions (with or without monomers in the aqueous phase), were prepared following the same procedure. First a polydisperse emulsion was obtained by manually incorporating $75 \mathrm{wt} \%$ of $0.1 \mathrm{M} \mathrm{NaCl}$ brine in IPM containing $10 \mathrm{wt} \%$ of PGPR with respect to the oil phase (for $100 \mathrm{~g}$ of reverse emulsion the composition was therefore as following: $75 \mathrm{~g}$ of solution containing $74.56 \mathrm{~g}$ of $\mathrm{H}_{2} \mathrm{O}$ in presence or not of monomer and $0.44 \mathrm{~g}$ of $\mathrm{NaCl}$ and $25 \mathrm{~g}$ of the oil phase composed of $22.5 \mathrm{~g}$ of IPM and 2.5 $\mathrm{g}$ of PGPR). This crude premixed emulsion was then sheared by a Couette cell composed of two concentric cylinders (commercialized by Ademtech). The inner cylinder of radius $\mathrm{Ri}=20 \mathrm{~mm}$ rotated with a speed of $150 \mathrm{rpm}$ and was spaced out (gap) from the outer static cylinder by $\mathrm{e}_{\text {couette }}=200 \mu \mathrm{m}$. Therefore, the applied shear rate was equal to $1560 \mathrm{~s}^{-1}$. The emulsion was injected in the device by a syringe pump. A Rayleigh instability developed leading to quasimonodisperse emulsions ${ }^{19,20}$

The resulting reverse emulsion drop size distribution has been measured using a preparation cell dedicated to oil continuous system. It is characterized by a mean diameter of $15 \mu \mathrm{m}(P=47 \%)$ as can be seen in Figure 1. 


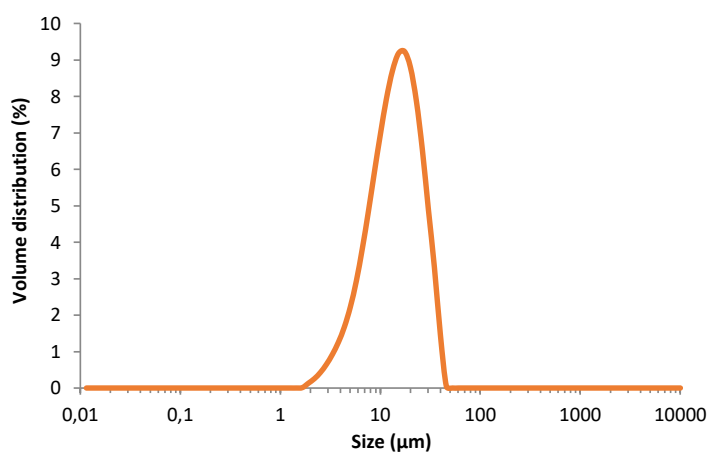

Figure 1: Drop size distribution of the reverse emulsion.

\section{4-Preparation of the double oil-in-water-in-oil emulsion}

Multiple oil-in-water-in-oil emulsions were prepared following a classical two-step procedure ${ }^{21}$ as more precisely described earlier ${ }^{16}$.

(a) Direct emulsion. First a polydisperse emulsion is obtained by manually incorporating the chosen A or B fragrance ( 90 wt\%) into an aqueous phase composed of salted water $(0.1 \mathrm{M}$ of $\mathrm{NaCl})$ and Tergitol 15-S-12 at $30 \mathrm{wt} \%$ with respect to the water phase. In other words, for $100 \mathrm{~g}$ of a direct emulsion, the composition was the following: $90 \mathrm{~g}$ of fragrance dispersed in an aqueous phase composed of $0.04 \mathrm{~g}$ of $\mathrm{NaCl}, 3 \mathrm{~g}$ of Tergitol $15-\mathrm{S}-12$ and $6.96 \mathrm{~g}$ of $\mathrm{H}_{2} \mathrm{O}$. The premixed emulsion was then sheared at $14280 \mathrm{~s}^{-1}$ thanks to the same Couette cell operating at $680 \mathrm{rpm}$ with a $100 \mu \mathrm{m}$ gap. The resulting direct emulsion is characterized by a mean diameter of 0.68 $\mu \mathrm{m}$ with a narrow size distribution $(\mathrm{Pd}=27 \%)$ as it can be seen Figure 2.
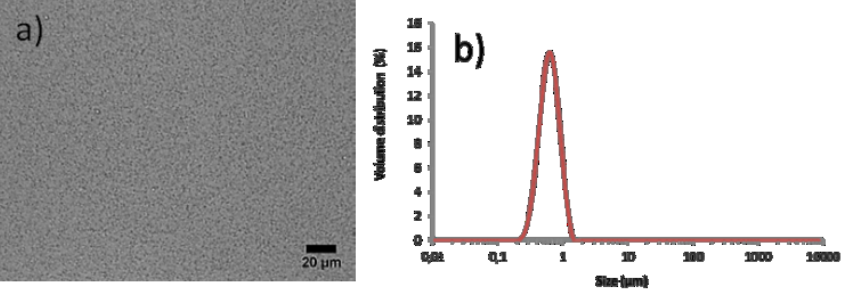

Figure 2: (a) Optical microscopy of direct emulsion and (b) corresponding droplet size distribution.

(b) Double emulsion. In a second step, the just described direct emulsion composed of either fragrance $A$ or fragrance $B$ was diluted by a factor ranging from 1.5 to 18 (leading to a fragrance fraction comprised between 5 and $60 \mathrm{wt} \%)$, depending on the targeted fraction of inner droplets, with an aqueous phase containing salted water $(0.1 \mathrm{M} \mathrm{NaCl})$ hydrophilic surfactant, initiator and monomer. The direct emulsion whose fragrance volume fraction $\left(\phi_{\mathrm{d}}\right)$ has been set by the dilution served as the dispersed phase of the reverse emulsion. It was progressively and manually incorporated into an oil phase containing IPM and $10 \mathrm{wt} \%$ of PGPR. The advantage of this two-step procedure that allows dilution of an initially highly concentrated direct emulsion ( $90 \mathrm{wt} \%$ ) is the possibility to tune both the mass fraction of inner droplets with respect to water drops $\left(\phi_{\mathrm{d}}\right)$ and the hydrophilic surfactant concentration in the aqueous phase \%HS.

\section{5-Interfacial tension measurement}

The interfacial tensions were measured with the weighted drop method. It consisted in shaping a drop of the heaviest phase at the end of a straight needle, which was immersed in the lightest phase in a beaker. The volume of the drop was slowly increased. When the weight of the drop became larger than the capillary force which retained the drop at the end of the needle, the drop detached and felt in the beaker placed on a precision balance. The drop's precise mass was measured. The operation was repeated at least 10 times. Just before detachment, the capillary force and the drop weight were equal so that the following relation applied:

$$
2 \pi r f \gamma=\Delta \rho \frac{m}{\rho} g
$$

where: $r$ is the radius of the needle $(r=0.3 \mathrm{~mm}), f$ is a corrective factor reflecting the complexity of the shape of the drop when it detaches (the expression of $f$ can be found in supporting information Equations S1), $m$ is the mass of the drop, $\rho$ the density of the heaviest liquid, $\Delta \rho$ the density difference between the two liquids and $\gamma$ the interfacial tension between them.

\section{6-Protocol of polymerization}

Polymerization of inverted and double emulsions were performed as described hereafter. KPS was used as an initiator. The total amount of salt (KPS and $\mathrm{NaCl}$ ) was fixed to $0.1 \mathrm{M}$ with respect to the aqueous phase.

(a) Polymerization of the inverted emulsion. All the emulsions were polymerized in the same way. The inverted emulsion was prepared as described previously with an aqueous phase containing both the monomer and the initiator.

The polymerization was proceeded in a flask with a magnetic stirrer in olive shape at a speed of $100 \mathrm{rpm}$ under nitrogen. The polymerization was initiated by increasing the temperature at $70^{\circ} \mathrm{C}$. The temperature and agitation were maintained during 24 $\mathrm{h}$ and the polymerization was stopped with the exposure to air. The polymer was washed with pentane (in twice its volume, a good solvent of all the reactants to eliminate) then isolated by successive centrifugation steps during 20 minutes at $10000 \mathrm{~g}(\mathrm{~g}$ being the gravity constant equal to $9.81 \mathrm{~m} \cdot \mathrm{s}^{-2}$ ). Then the polymer was dried during $12 \mathrm{~h}$ at $50^{\circ} \mathrm{C}$ under vacuum. The conversion of the monomer was obtained by gravimetric measurement thanks to the following relation (4):

$$
\text { Conversion }=\frac{\mathrm{m}_{\text {obtained polymer }}}{\% \text { monomer } * \mathrm{~m}_{\text {collect }}}
$$

$m_{\text {obtainedpolymer }}$ is the weight of polymer obtained after purification and drying subtracting the salt. $\mathrm{m}_{\text {collect }}$ represents the quantity of polymerized medium weighted before the 
purification and \%monomer represents the weight percentage of monomer initially present in the sample.

\section{Synthesis of POEGMA}

The reverse emulsion was prepared with this monomer with a concentration of $25 \mathrm{wt} \%$ of monomer in the aqueous phase. In other words, for $100 \mathrm{~g}$ of sample, the water phase was composed of $18.75 \mathrm{~g}$ of OEGMA, $0.04 \mathrm{~g}$ of KPS, $0.42 \mathrm{~g}$ of $\mathrm{NaCl}$ and $55.79 \mathrm{~g}$ of $\mathrm{H}_{2} \mathrm{O}$. This water phase represented $75 \mathrm{wt} \%$ of the total sample $(75 \mathrm{~g})$. The $25 \mathrm{~g}$ of oil phase was composed of 22.5 $\mathrm{g}$ of IPM and $2.5 \mathrm{~g}$ (10 wt\%) of PGPR.

\section{Synthesis of PMAM}

The reverse emulsion was prepared with this monomer with a concentration of $15 \mathrm{wt} \%$ of monomer in the aqueous phase.

In other words, for $100 \mathrm{~g}$ of sample, the aqueous phase was composed of $11.25 \mathrm{~g}$ of MAM, $0.1 \mathrm{~g}$ of KPS, $0.37 \mathrm{~g}$ of $\mathrm{NaCl}$ and $63.26 \mathrm{~g}$ of $\mathrm{H}_{2} \mathrm{O}$. This aqueous phase represented $75 \mathrm{wt} \%$ of the total sample $(75 \mathrm{~g})$. The $25 \mathrm{~g}$ of oil phase was composed of 22.5 $\mathrm{g}$ of IPM and $2.5 \mathrm{~g}$ (10 wt\%) of PGPR.

\section{Synthesis of PHEMA}

The reverse emulsion was prepared with this monomer with a concentration of $25 \mathrm{wt} \%$ and $50 \mathrm{wt} \%$ of monomer in the aqueous phase.

In other words, for $25 \mathrm{wt} \%$ of monomer, for $100 \mathrm{~g}$ of sample, the aqueous phase was composed of $18.75 \mathrm{~g}$ of HEMA, $0.09 \mathrm{~g}$ of KPS, $0.40 \mathrm{~g}$ of $\mathrm{NaCl}$ and $55.76 \mathrm{~g}$ of $\mathrm{H}_{2} \mathrm{O}$. This aqueous phase represented $75 \mathrm{wt} \%$ of the total sample $(75 \mathrm{~g})$. The $25 \mathrm{~g}$ of oil phase was composed of $22.5 \mathrm{~g}$ of IPM and $2.5 \mathrm{~g}$ (10 wt\%) of PGPR.

For 50 wt\% of monomer, if we considered $100 \mathrm{~g}$ of sample, the water phase was composed of $37.5 \mathrm{~g}$ of HEMA, $0.18 \mathrm{~g}$ of KPS, $0.36 \mathrm{~g}$ of $\mathrm{NaCl}$ and $36.96 \mathrm{~g}$ of $\mathrm{H}_{2} \mathrm{O}$. This water phase represented $75 \mathrm{wt} \%$ of the total sample $(75 \mathrm{~g})$. The $25 \mathrm{~g}$ of oil phase was composed of $22.5 \mathrm{~g}$ of IPM and $2.5 \mathrm{~g}(10 \mathrm{wt} \%)$ of PGPR.

(b) Polymerization of the oil-in-water-in-oil emulsion. The composition is the same as previously described apart that a portion of the water phase was replaced by fragrance. In the following the double emulsion was composed of $\phi_{\mathrm{d}}=20 \mathrm{wt} \%$ of fragrance in the water globules, themselves corresponding to $75 \mathrm{wt} \%$ of the total sample. The composition of surfactants was kept constant: $\%$ Hs $=7.5 \mathrm{wt} \%$ with respect to the aqueous phase and \%LS=10 wt\% with respect to the outer IPM (\%Hs, \%Ls, mass \% of hydrophilic and lipophilic surfactants respectively. All the multiple emulsions were polymerized with the same protocol than the one described for inverted emulsion. The only difference was the purification. The washing was proceeded with ethanol instead of pentane because the residual reactants and fragrance to eliminate are very soluble in ethanol. After this step, the solid obtained was lyophilized. The conversion was also obtained by gravimetric measurement thanks to the following relation (5):

$$
\text { Conversion }=\frac{\mathrm{m}_{\text {obtainedpolymer } * \%_{\text {solidTGA }}}}{\%_{\text {monomer }} * \mathrm{~m}_{\text {collect }}}
$$

$\mathrm{m}_{\text {obtainedpolymer }}$ is the weight of polymer obtained after purification and drying subtracting the salt. $\mathrm{m}_{\text {collect }}$ represents the quantity of polymerized medium weighted before the purification. \%monomer represents the initial weight percentage of monomer present initially in the sample and \%solidtGA represents the quantity of polymer in the sample after the elimination of remaining water despite lyophilization. It was obtained by TGA.

\section{Synthesis of PMAM}

The monomer was added to the aqueous phase at a concentration of $15 \mathrm{wt} \%$ with respect to the globules (fragrance add aqueous phase) corresponding to $11.25 \mathrm{~g}$ of MAM and 0.1 $\mathrm{g}$ of KPS for a total sample of $100 \mathrm{~g}$. The oil phase was unchanged.

\section{Synthesis of POEGMA}

The double emulsion was formulated with $25 \mathrm{wt} \%$ of monomer in the globules. In other words, if we considered $100 \mathrm{~g}$ of sample, it contained $18.75 \mathrm{~g}$ of OEGMA, $0.04 \mathrm{~g}$ of KPS. Again the oil phase was unchanged.

\section{(c) Polymer characterization}

The polymers were analyzed by Differential Scanning Calorimetry (DSC) with a DSC Q100 RCS apparatus. The protocol was the following: first cycle: heating from $-80^{\circ} \mathrm{C}$ to $120^{\circ} \mathrm{C}$ with a temperature ramp of $10^{\circ} \mathrm{C} / \mathrm{min}$ followed by cooling down to $80^{\circ} \mathrm{C}$ with a temperature ramp of $10^{\circ} \mathrm{C} / \mathrm{min}$. The second cycle started at $-80^{\circ} \mathrm{C}$ up to $230^{\circ} \mathrm{C}$ with a ramp of $10^{\circ} \mathrm{C} / \mathrm{min}$ followed by a cooling down to $-50^{\circ} \mathrm{C}$ with the same temperature ramp.

The polymer was also characterized by TGA (Thermogravimetric Analysis) with an apparatus TGA Q500 commercialized by TA instruments. The sample was heated up to $600^{\circ} \mathrm{C}$ with a ramp of $10^{\circ} \mathrm{C} \cdot \mathrm{mn}^{-1}$ under air.

\section{Results and Discussion}

The double emulsion was prepared with a 2 step process as described previously ${ }^{16,20,21}$ in section $4 \mathrm{~b}$ (Scheme 1). First of all, the direct emulsion was produced by emulsifying the inner lipophilic fragrance in a salted water phase in presence of the hydrophilic surfactant (Tergitol 15-S-12). After dilution of this direct emulsion, the second step consisted in dispersing it in the second oil phase (IPM) containing the lipophilic surfactant (PGPR). The obtained multiple emulsions were of the oil-in-water-in-oil type. Tergitol 15-S12 and PGPR have been respectively selected in agreement with a previous study ${ }^{16}$ for their ability to produce a direct emulsion with a mean droplet size close to $1 \mu \mathrm{m}$ and a reverse emulsion with a mean globule size of $15 \mu \mathrm{m}$ respectively (see Figure 1 and Figure 2). IPM was chosen as the oil continuous phase because it is commonly used in cosmetics and perfumery. The sodium chloride was incorporated in the water phase to increase the reverse emulsion stability. 


\section{ARTICLE}
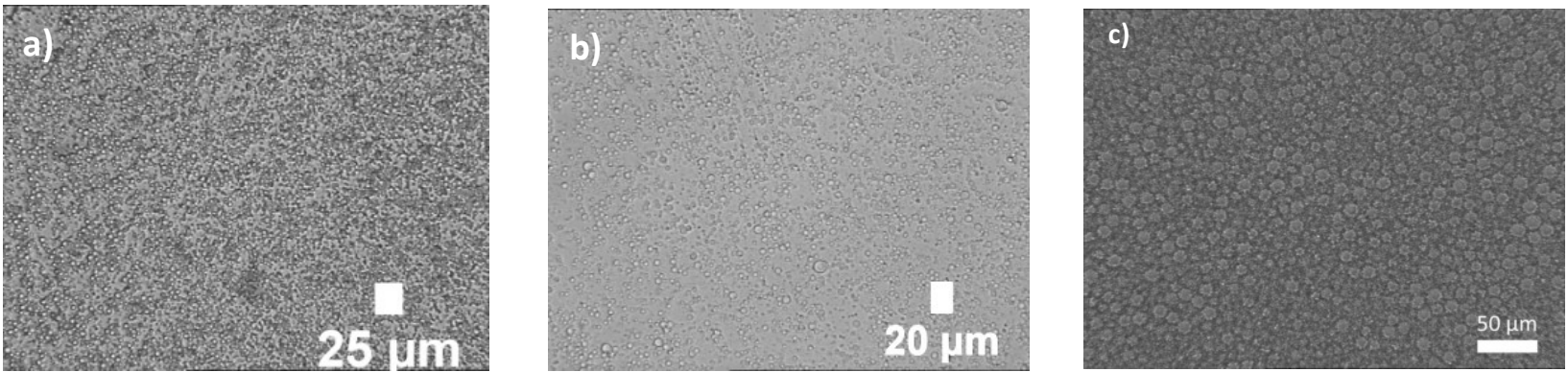

Figure 3: Observation by optical microscopy of the reverse emulsion prepared with (a) $25 \mathrm{wt} \%$ (b) $50 \mathrm{wt} \%$ and (c) 0 wt\% of HEMA in the water phase.

To determine whether monomers may destabilize the double emulsion, we first prepared reverse emulsions in presence of monomer and initiator and studied the polymerization of this simple emulsion alone. Then, the formulation of the double emulsion with the monomers was tested to determine the best appropriate composition.

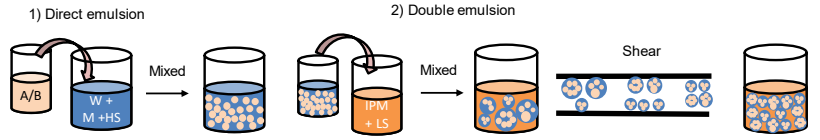

Scheme 1: Fabrication of double emulsion

Finally, the polymerization of the chosen double emulsion was performed for each monomer.

\section{Preparation and polymerization of simple reverse emulsions}

Three monomers chosen for their hydrophilicity and biocompatibility have been evaluated: HEMA, OEGMA, MAM The study of the reverse emulsions was divided in two parts. First of all, the formulation of the reverse emulsions with the different monomers was proceeded in order to identify the most interesting ones in terms of stability and emulsions drop size. The second part is the polymerization with the selected monomers.

(a) Formulation of the reverse emulsions. The different reverse emulsions were prepared by adding the monomer in the aqueous phase at different concentrations. The first studied monomer was HEMA. The preparation of the emulsion was performed for two concentrations (25 wt\% and $50 \mathrm{wt} \%$ of HEMA). It turned out that the introduction of the monomer inside the aqueous phase induced a large decrease of the droplet size as it can be observed in Figures 3 and S1, $4.9 \mu \mathrm{m}$ and $7.1 \mu \mathrm{m}$ for $25 \mathrm{wt} \%$ and $50 \mathrm{wt} \%$ of HEMA in the water phase respectively to be compared with $15 \mu \mathrm{m}$ for the reverse emulsion without HEMA).
Such a size decrease indicates that HEMA likely exhibits interfacial properties. To evidence it, interfacial measurements were performed. The interfacial tension between water and IPM was measured and compared to the one obtained between a water phase containing $25 \mathrm{wt} \%$ of HEMA and IPM. The addition of the monomer in the water phase induced a large decrease of the interfacial tension $\left(38.1 \mathrm{mN} . \mathrm{m}^{-1}\right.$ without monomer to be compared with $5.8 \mathrm{mN} \cdot \mathrm{m}^{-1}$ with monomer) evidencing the interfacial affinity of HEMA and therefore its migration to the interface in agreement with the emulsion drop diameter decrease. As we targeted drops around $15 \mu \mathrm{m}$ to be able to encapsulate the direct emulsion, work with this HEMA monomer was not continued.

As far as OEGMA and MAM are concerned, the preparation of the reverse emulsion at $25 \mathrm{wt} \%$ and $15 \mathrm{wt} \%$ (due to the relative solubility of MAM in water of $202 \mathrm{g.L}^{-1}$ ) respectively, in the aqueous phase gave emulsions with appropriate droplet diameters as shown in Figure 4a (diameters of $8.2 \mu \mathrm{m}$ and $9.2 \mu \mathrm{m}$ for OEGMA and MAM respectively).

Moreover, as can be seen from Figure $4 \mathrm{~b}$, the emulsions were stable with no size evolution for more than 20 hours.

OEGMA and MAM were hence two appropriate candidates to study the reverse emulsion polymerization.

(b) Polymerization of the reverse emulsions. Free radical polymerization of the reverse emulsion prepared with MAM and with OEGMA will be considered in this part. These polymerizations were initiated by KPS. They were performed in flask with magnetic stirring for $24 \mathrm{~h}$ and were quenched by exposure to air.

\section{Synthesis of POEGMA}

After the polymerization, the obtained suspension exhibited a particle diameter equal to $7.8 \mu \mathrm{m}$ close to the initial emulsion drop size $(8.2 \mu \mathrm{m})$. This means that no aggregation or 
coagulation occurred during the polymerization. Due to the

very
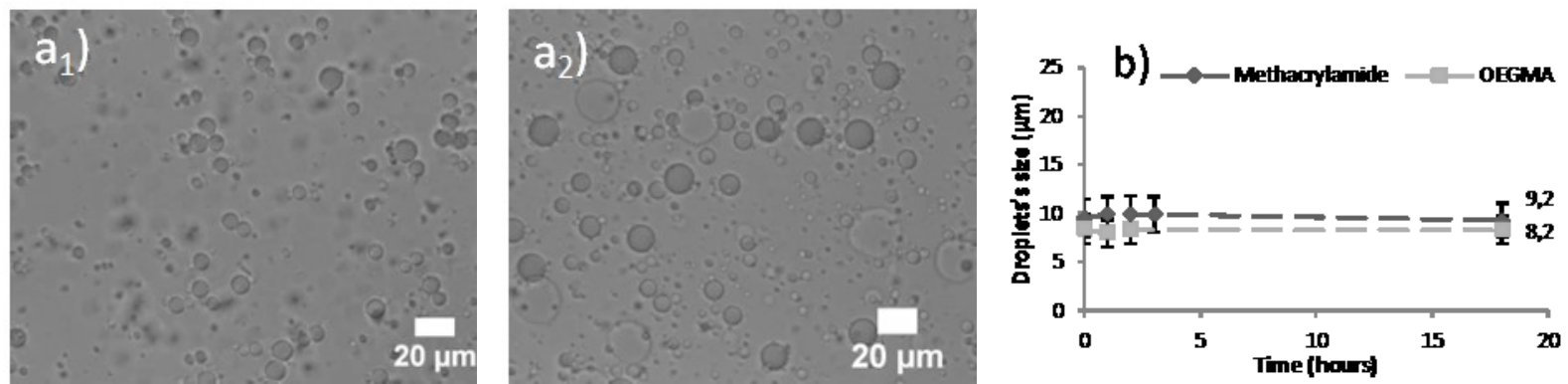

Figure 4: (a) Observation by optical microscopy of reverse emulsion prepared with $\left(a_{1}\right) 25$ wt\% of OEGMA $\left(a_{2}\right) 15$ wt $\%$ of MAM. (b) emulsion drop diameter vs time for the two previous emulsions. The error bars represent the diameter polydispersity of the drops in the emulsion.

low solubility of POEGMA in usual solvents available for NMR or SEC, the polymer was analyzed by DSC (Figure 5).

As it can be seen on Figure 5 , the synthesized polymer exhibits a glass temperature $\left(\mathrm{T}_{\mathrm{g}}=-53^{\circ} \mathrm{C}\right)$, a crystallization temperature $\left(T_{c}=-8^{\circ} \mathrm{C}\right)$ and a melting point $\left(T_{m}=7^{\circ} \mathrm{C}\right)$ characteristic of polyethylene glycol (PEG) chains. Compared with those of the OEGMA macromonomer, a significant increase of glass temperature $\left(T_{g}=-61^{\circ} \mathrm{C}\right)$ was observed (Supporting data, Figure S2). This behavior can be explained by the decrease of the mobility of the OEGMA grafted chains by comparison with free OEGMA chains attesting that the polymerization occurred.

The OEGMA conversion was close to $100 \%$ (91\%).

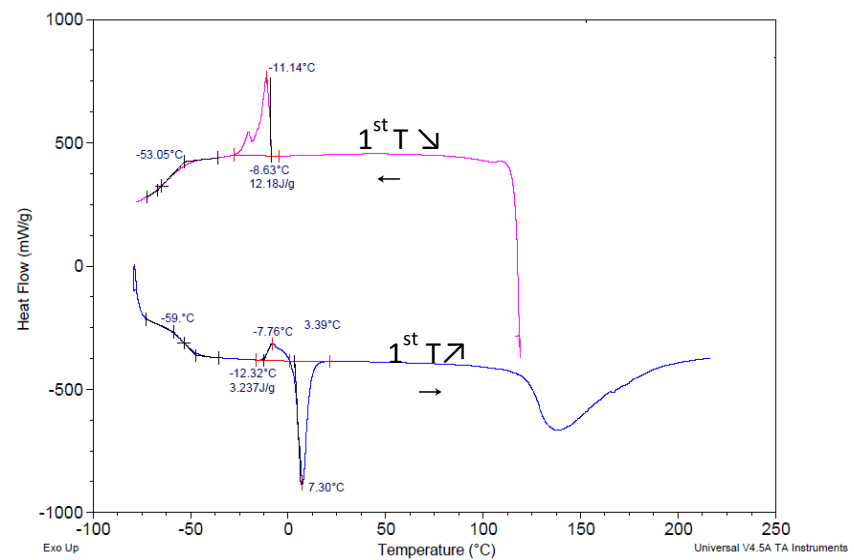

Figure 5: DSC thermogram of the polymerized reverse emulsion prepared with $25 \%$ of OEGMA

\section{Synthesis of PMAM}

After polymerization, the obtained suspension is analyzed by optical microscopy and size-distribution analysis. The presence of two main populations is evidenced (Figure 6). The population with a diameter of about $40 \mu \mathrm{m}$ corresponds to aggregates of several polymerized drops that are also visible by optical microscopy. However, these aggregates are no coagulum since the particles can move with respect to each other when a shear is applied between the two glass slides. The population, with a diameter of about $10 \mu \mathrm{m}$ corresponds to isolated polymerized drops (same diameter as drops before the polymerization).
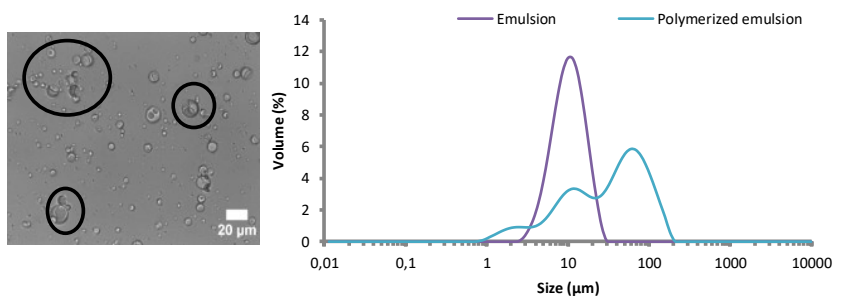

Figure 6: Left: optical microscopy of the polymerized emulsion containing $15 \mathrm{w} \%$ of MAM. The black circles enclose the aggregates. Right: particle size distribution before and after polymerization.

DSC analysis was proceeded. Three heating/cooling cycles were performed (see Figure 7). During the first cycle, a transition at $50-150^{\circ} \mathrm{C}$ was visible but this one disappeared during the second and third cycles. This endothermic change is probably caused by the evaporation of residual water. The endothermic change at a temperature above $150^{\circ} \mathrm{C}$ could be attributed to the thermal reaction of amide groups to convert to imide groups ${ }^{22}$. It can also be attributed to physical crosslinking due to hydrogen bonding formation ${ }^{23}$. In addition, the physical crosslinking of PMAM chains was validated by the non-solubility of the polymer except in strong acids known to be able to disrupt hydrogen bonds.

The MAM conversion obtained by gravimetric measurement was $64 \%$. 


\section{ARTICLE}

Table 2: Summary table of the different double emulsion formulations with the addition of OEGMA No double emulsion could be obtained Double emulsion, Not proceeded (NP)

\begin{tabular}{|c|c|c|c|c|c|c|}
\hline $\begin{array}{l}\text { O/W/O composition } \\
\phi_{\mathrm{d}} / \% \mathrm{HS}(\mathrm{wt} \% / \mathrm{wt} \%)\end{array}$ & $45 / 20$ & $30 / 20$ & $30 / 15$ & $30 / 7.5$ & $20 / 10$ & $20 / 7.5$ \\
\hline $25 \mathrm{w} \%$ of OEGMA & & & & & & \\
\hline $50 \mathrm{w} \%$ of OEGMA & NP & NP & NP & & NP & \\
\hline
\end{tabular}

On the basis of these positive results achieved with simple reverse emulsions, we decided to focus on these two monomers for the rest of the study.

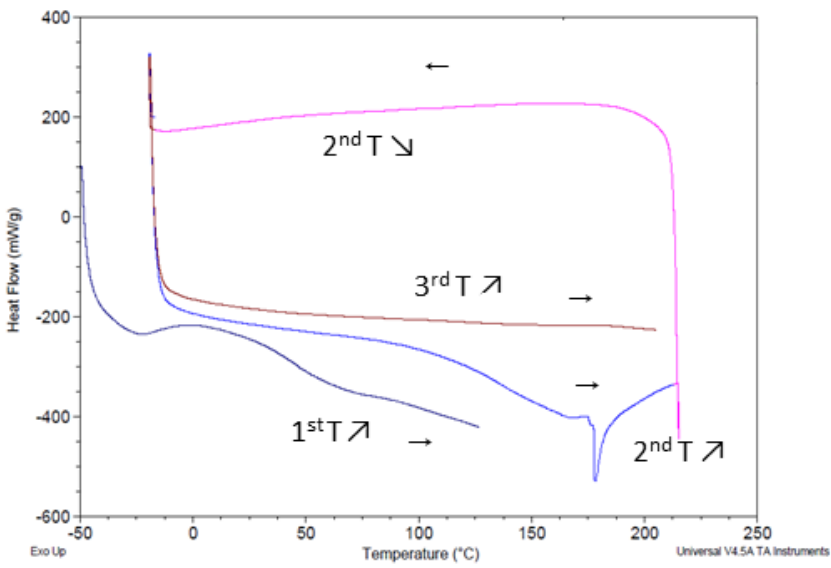

Figure 7: Thermogram obtained by DSC of the polymer resulting of the polymerization of the reverse emulsion formulated with $15 \mathrm{w} \%$ of MAM in the aqueous phase.

\section{Study of the double emulsion formulated with the monomer}

The formulation of the double emulsion has been done in agreement with results published previously ${ }^{16} ; \phi_{d}$ wt\% of droplets inside the water phase and \% $\%_{\text {HS }}$ of hydrophilic surfactant. In other words, a part of the intermediate aqueous phase has been replaced by droplets fragrance and $\mathrm{HS}$.

First, the effect of the monomer addition in the water phase, on the size and stability of double emulsion, has been investigated in the particular case of the fragrance $B$.

Then, the system with the best repartition of droplets inside the globules was fixed and polymerized with the two monomers and fragrances $A$ and $B$.

(a) Formulation of the double emulsions.

Formulations with OEGMA
Six different compositions, for which only multiple drops could be obtained $^{16}$, have been selected. OEGMA ( $25 \mathrm{wt} \%$ and $50 \mathrm{wt} \%$ ) was added in the water phase of these formulations (Table 2). Only three compositions allowed obtaining double emulsion (Figure 8). Moreover, even if we succeeded in formulating emulsions with 25 wt\% of monomer, we failed with 50 wt\%. This is probably because this monomer exhibits interfacial properties $\left(8.4 \mathrm{mN} \cdot \mathrm{m}^{-1}\right.$ for interfacial tension of OEGMA in water and IPM) showing that it migrates to the interface as HEMA did but to a lower extent ( 5.8 $\mathrm{mN} \cdot \mathrm{m}^{-1}$ for the interfacial tension of HEMA in water against IPM). The lowered migration of OEGMA to the interface compared to HEMA could be explained by larger size of OEGMA. Interfacial properties of OEGMA have also been observed by Kasantsev ${ }^{24}$.

To conclude, three different compositions were possible with the OEGMA. Among these compositions, we have chosen the one with $20 \mathrm{wt} \%$ of droplets inside the water phase and $7.5 \mathrm{wt} \%$ of Tergitol 15-S-12 (Figure 8a).

\section{Formulation with MAM}

We chose to keep the same emulsion composition as for OEGMA, e. g.20 wt\% of droplets inside the water phase and $7.5 \mathrm{wt} \%$ of Tergitol 15-S-12 and to incorporate MAM (Figure 9).

As observed in Figure 9, the obtained emulsion was only composed of globules homogeneously filled with droplets (only a multiple emulsion).

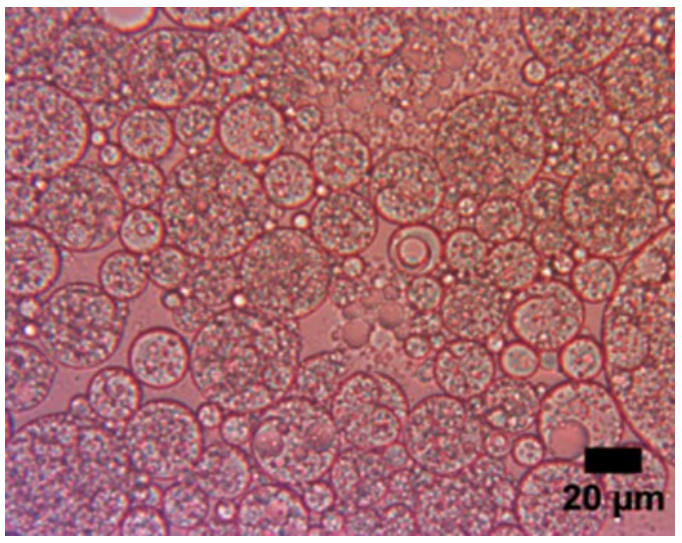


Figure 9: Observation by optical microscopy of the double emulsion at $\phi_{d}=20 \mathrm{wt} \%$ and $\% \mathrm{HS}=7.5 \mathrm{wt} \%$ with $15 \mathrm{wt} \%$ of MAM inside the water phase.

To conclude this part, addition of monomers in the double emulsion narrows the composition domains where double emulsions can be prepared.
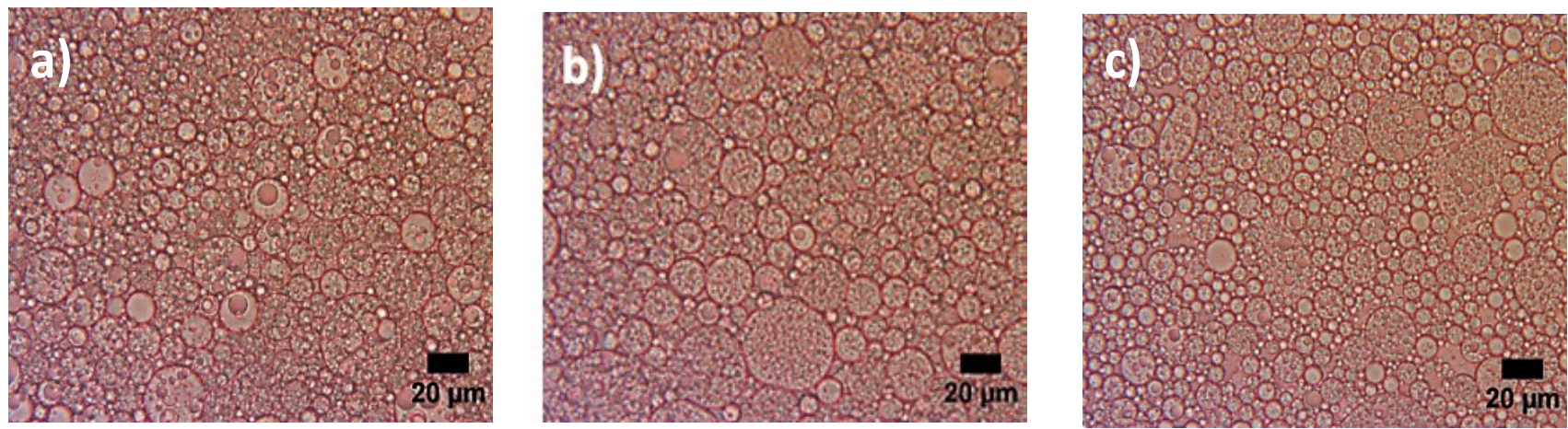

Figure 8: Observation by optical microscopy of the different formulations which allowed obtaining double emulsions containing $25 \mathrm{wt} \%$ of OEGMA a) $\phi_{\mathrm{d}}=20 \mathrm{wt} \%$ and $\%_{\mathrm{HS}}=7.5 \mathrm{wt} \%$ b) $\phi_{\mathrm{d}}=20 \mathrm{wt} \%$ and $\%$ Hs $=10 \mathrm{wt} \%$ c) $\phi_{\mathrm{d}}=30 \mathrm{wt} \%$ and $\%$ Hs $=7.5 \mathrm{wt} \%$.

(b) Polymerization of the chosen systems. To limit the number of polymerizations and to focus on the effect of the monomer, we selected one emulsion composition for the rest of the study: $\phi_{d}=20$ $w t \%, \%{ }_{H S}=7.5 w t \%$, total salt concentration ( $\mathrm{NaCl}$ and $\mathrm{KPS}$ ) equal to $0.1 \mathrm{M}, \Phi_{\mathrm{G}}=75 \%$ and $\mathrm{C}_{\mathrm{PGPR}}=10 \mathrm{wt} \%$ with respect to external oil.

Three different systems were polymerized with the composition and the protocol described in the experimental part:

1) Emulsion with 25 wt\% of OEGMA and the fragrance $B$ (OEGMA/B)

2) Emulsion with 25 wt\% of OEGMA and the fragrance $A$ (OEGMA/A)

3) Emulsion with $15 w t \%$ of MAM and the fragrance $B$ (MAM/B)

For the three systems, a gel was obtained because of the high concentration of globules (75 wt\%). In order to characterize these globules, we attempted to disperse them in water (good solvent of the polymer) and in IPM (continuous phase of the initial double emulsion). Surprisingly, we noticed that none of the polymerized double emulsions was dispersible in IPM. IPM is thus not a good solvent of the resulting polymer and caused a polymer collapse as it can be observed in the Supporting Data Figure S3.

On the other hand, the three systems can be dispersed in water, the polymerized multiple drops can be then observed by optical microscopy. It is explained by the fact that water is a good solvent of
POEGMA and PMAM, it likely swells the polymer particles (not enough to be observable) and separates them, which allows the observation of the better dispersed compartmentalized particles (Figure 10).

The DSC thermograms (Supporting data Figure S4) were very similar to those obtained for the polymerized reverse emulsions and confirmed the polymerization step.

The monomer conversions were: $35 \%, 6 \%$ and $18 \%$ for OEGMA/B, OEGMA/A and MAM/B respectively. Even if these values are relatively low, they show that polymerization of the intermediate phase of double emulsion was possible. One should keep in mind that none of these three polymerizations has been optimized. It is interesting to note the large difference between OEGMA/B and OEGMA/A in term of conversion whereas the only variance was the used fragrance. This is likely due to the difference of interfacial behavior between the two fragrances. Indeed, the interfacial tension between water and fragrance $A$ was significantly smaller than with fragrance $B$ (Table 1 ). The energy required to form an interface between the fragrance $A$ and water is lower than the one required to form an interface between fragrance $B$ and water even if the Log $P$ (estimated with octanol) of both fragrances are close. In other words, the molecules of fragrance $A$ are more likely to migrate towards the interface than the molecules of fragrance $B$.
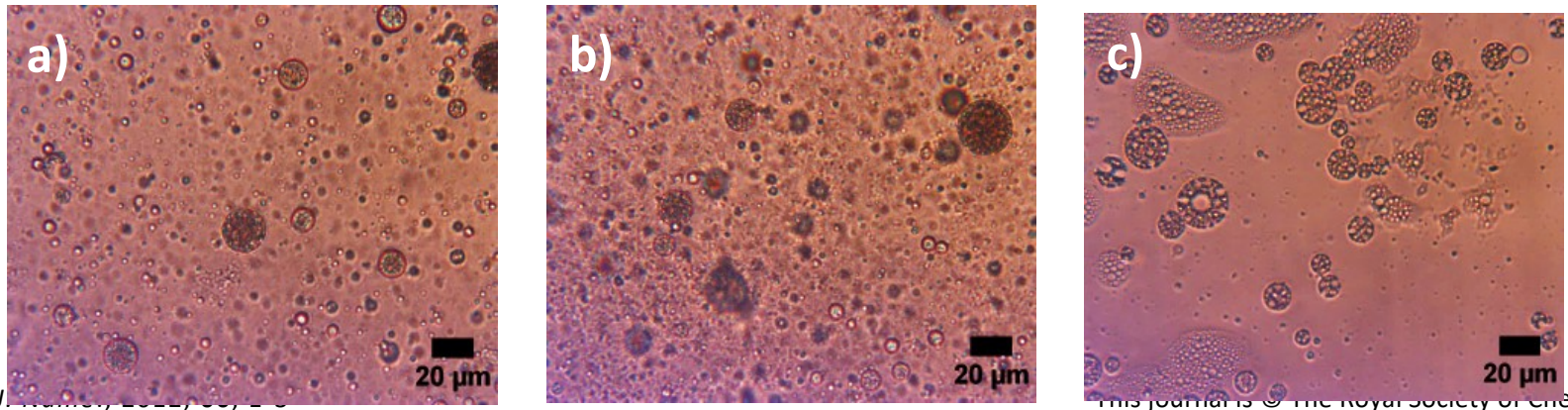


\section{Supporting information}

Figure 10: Observation by optical microscopy of a) OEGMA/B b) OEGMA/A and c) MAM/B after polymerization and dispersion in water.

This migration can provoke interaction between the fragrance molecules and the polymerization and can reduce the conversion of the polymerization.

\section{Conclusions}

The formulation of reverse emulsion with hydrophilic monomers allowed evidencing that not only the monomer should be hydrophilic but also it should not have interfacial properties to make it possible to prepare a double emulsion with a difference in size of droplet and globule large enough to allow the encapsulation of the droplets.

For example, we have shown that the presence of HEMA reduces too significantly the size of the globules because of its ability to migrate to the interface. This size reduction prevents the encapsulation of fragrance droplets. Two different monomers that do not alter the complex double emulsion structure were selected: OEGMA and MAM. When these two monomers are present in the dispersed phase of a reverse $\mathrm{W} / \mathrm{O}$ emulsion, they can be polymerized with a conversion higher than $60 \%$. The monomer incorporation leads to narrower accessible composition domains for the double emulsions. However, for a unique double emulsion composition: $\phi_{d}=20 \mathrm{wt} \%$, $\%{ }_{\mathrm{HS}}=7.5 \mathrm{wt} \%, \mathrm{NaCl}$ and $\mathrm{KPS}=0.1 \mathrm{M}$ in the globules and $\Phi_{\mathrm{G}}=75 \%$ and $C_{P G P R}=10 w t \%$ with respect to external oil, compartmentalized capsules can be obtained for both fragrances with identical LogP values and despite their different interfacial behaviors. Indeed, fragrance $A$ is composed of molecules that are more likely to migrate to the interface possibly hampering the polymerization. Consequently, the conversion is much lower for fragrance $A$ than for fragrance $B$ (for OEGMA: $6 \%$ of conversion for fragrance $A$ versus $35 \%$ for fragrance $B)$. For fragrance $B$, a lower conversion was obtained for MAM (18\%) than OEGMA (35\%). However, MAM remains an interesting monomer for future applications because of its physical crosslinking ability.

\section{Conflicts of interest}

There is no conflict of interest to declare for any of the authors.

\section{Acknowledgements}

The authors would like to thank T.E.P.L and ANRT (contract number $N^{\circ}$ 2014/1309) for financial support with contract number: 2014/1309.
Composition of A \& B fragrances.

Equations S1: Formula of corrective factors.

Figure S1: Comparison of the globules'size of the reverse emulsion prepared with $25 \mathrm{w} \%$ and $50 \mathrm{w} \%$ of HEMA in the water phase

Figure S2: DSC of OEGMA macromonomer polymerized

Figure S3: Microphotographs of polymerized OEGMA/B, OEGMA/A and MAM/B O/W/O emulsions in IPM.

Figure S4: DSC of OEGMA/B, OEGMA/A and MAM/B O/W/O.

\section{References}

1 H. N. Yow and A. F. Routh, Soft Matter, 2006, 2, 940.

2 R. Dubey, T.C. Shami and K.U.Bhasker, Defence Science Journal, 2009, 59, 82.

3 Green, B.K. and S. Lowell, Oil-containing microscopic capsules and method of making them. US Patent 28004571957.

4 C. Pinto Reis, R. J. Neufeld, A. J. Ribeiro, and F. Veiga, Nanomedicine: Nanotechnology, Biology and Medicine, 2006, 2, 8.

5 J. Malanovic, V. Manojilovic, S. Levic, N. Rajic, V. Nedovic and B. Burgarski, Sensors, 2010, 10, 901.

6 K. Hirech, S. Payan, G. Carnelle, L. Brujes and J. Legrand, Powder Technol., 2003, 130, 324.

7 G. León, N. Paret, P. Fankhauser, D. Grenno, P. Erni, L. Ouali and D. L. Berthier, RSC Adv., 2017, 7, 18962.

8 S. Bône, C. Vautrin, V. Barbesant, S. Truchon, I. Harrison and C. Geffroy, CHIMIA International Journal for Chemistry, 2011, 65, 177.

9 A. P. R. Johnston, C. Cortez, A. S. Angelatos and F. Caruso, Current Opinion in Colloid \& Interface Science, 2006, 11, 203.

10 S. Le Tirilly, C. Tregouët, S. Bône, C. Geffroy, G. Fuller, N. Pantoustier, P. Perrin and C. Monteux, ACS Macro Letters, 2015, 4, 25.

11 I. Gürsel and V. Hasirci, Journal of Microencapsulation, 1995, 12, 185.

12 M. A. Teixeira, O. Rodríguez, S. Rodrigues, I. Martins and A. E. Rodrigues, AIChE Journal, 2012, 58, 1939.

13 M. Jacquemond, N. Jeckelmann, L. Ouali and O. P. Haefliger, Journal of Applied Polymer Science, 2009, 114, 3074.

14 E. Jahns, D. Boeckh, W. Bertleff, and P. Neumann, Microcapsule preparations and detergents and cleaning agents containing microcapsules. US Patent 2003/012.5222 A1

15 J. Berg, D. Sundberg and B. Kronberg, Journal of Microencapsulation, 1989, 6, 327.

16 M. Stasse, T. Ribaut, O. Anthony, V. Héroguez and V. Schmitt, Langmuir, (submitted). 
17 C. Laane, S. Boeren, K. Vos and C. Veeger, Biotechnology and Bioengineering, 1987, 30, 81.

18 M. Nollet, M. Mercé, E. Laurichesse, A. Pezon, O. Soubabère, S. Besse and V. Schmitt, Soft Matter, 2016, 12, 3412.

19 C. Mabille, V. Schmitt, P. Gorria, F. Leal Calderon, V. Faye, B. Deminière and J. Bibette, Langmuir, 2000, 16, 422.

20 C. Mabille, F. Leal-Calderon, J. Bibette and V. Schmitt, Europhysics Letters (EPL), 2003, 61, 708.

21 C. Goubault, K. Pays, D. Olea, P. Gorria, J. Bibette, V. Schmitt and F. Leal-Calderon, Langmuir, 2001, 17, 5184.

22 S. Matsuda, S. Tsuchiya, M. Honma, K. Hasegawa, G. Nagamatsu and T. Asano, Polymer Engineering and Science, 1977, 17, 410.

23 S.-W. Kuo, H.-C. Kao and F.-C. Chang, Polymer, 2003, 44, 6873.

24 O. A. Kazantsev, D. M. Kamorin, D. V. Orekhov and A. P. Sivokhin, Designed Monomers and Polymers, 2015, 18, 378. 